\title{
Energy level alignment and interactive spin polarization at organic/ferromagnetic metal interfaces for organic spintronics
}

\author{
Zhengyi Sun, Yiqiang Zhan, Shengwei Shi and Mats Fahlman
}

\section{Linköping University Post Print}

\section{Tweet}

N.B.: When citing this work, cite the original article.

Original Publication:

Zhengyi Sun, Yiqiang Zhan, Shengwei Shi and Mats Fahlman, Energy level alignment and interactive spin polarization at organic/ferromagnetic metal interfaces for organic spintronics, 2014, Organic electronics, (15), 9, 1951-1957.

http://dx.doi.org/10.1016/j.orgel.2014.05.021

Copyright: Elsevier http://www.elsevier.com/

Postprint available at: Linköping University Electronic Press http://urn.kb.se/resolve?urn=urn:nbn:se:liu:diva-108494 


\title{
Energy level alignment and interactive spin polarization at or- ganic/ferromagnetic metal interfaces for organic spintronics
}

\author{
Zhengyi Sun a,*, Yiqiang Zhan b,*, Shengwei Shi a, Mats Fahlman a \\ a Department of Physics, Chemistry and Biology (IFM), Linköping University, SE-581 83 Linköping, Sweden \\ b State Key Laboratory of ASIC and System, Department of Microelectronics, SIST, Fudan University, Shanghai 200433, \\ China
}

KEYWORDS: organic spintronics, photoemission spectroscopy, X-ray magnetic circular dichroism, spin polarization, organic-metal interface

\begin{abstract}
Energy level alignment and spin polarization at tetracyanoquinodimethane/Fe and acridine orange base $/ \mathrm{Fe}$ interfaces are investigated by means of photoelectron spectroscopy and X-ray magnetic circular dichroism (XMCD), respectively, to explore their potential application in organic spintronics. Interface dipoles are observed at both hybrid interfaces, and the work function of $\mathrm{Fe}$ is increased by $0.7 \mathrm{eV}$ for the tetracyanoquinodimethane (TCNQ) case, while it is decreased by $1.2 \mathrm{eV}$ for the acridine orange base (AOB) case. According to XMCD results, TCNQ molecule has little influence on the spin polarization of Fe surface. In contrast, AOB molecule reduces the interfacial spin polarization of Fe significantly. Induced spin polarization of the two organic molecules at the interfaces is not observed. The results reveal the necessity of investigating the magnetic property changes of both the OSC and the FM during the process of energy level alignment engineering.
\end{abstract}

\section{Introduction}

Studies on spin-related phenomenon in organic semiconductors (OSCs), known as "organic spintronics", have been carried out and have attracted increasing attention over the last decade. ${ }^{1-3}$ There are two main types of organic spintronic effects under study and development. One is organic magnetoresistance (OMAR), ${ }^{3}$ usually accompanied by magnetoluminescence. ${ }^{4,5}$ It occurs in conventional OSCs electronic devices with non-magnetic electrodes. This type of intrinsic magnetoresistance is believed to be connected to various excited states in OSCs, ${ }^{6}$ and, as evident from the lack of magnetic electrodes, has nothing to do with spinpolarized charge injection. The other one is based on the so-called spin valve that has a layered structure comprising ferromagnetic (FM) electrodes and a non-magnetic spacer layer, whose electrical resistance depends on the spinpolarization of the injected carriers passing through the device. ${ }^{2,7,8}$ In spin valves with an organic spacer layer, OSCs are used for the carrier spin transport as they consist mainly of atoms with low atomic number $\mathrm{Z}$, leading to a low spin-orbit coupling and thus to extremely long spin relaxation times. ${ }^{9,10}$ Injection of spin-polarized electrons from ferromagnetic electrode into an organic interlayer may be affected adversely, however, by a mismatch of conductivity between the FM metal and semiconductor for Ohmiclike contacts. ${ }^{11}$ For both giant magnetoresistance ${ }^{2,7,12}$ and tunneling magnetoresistance $e^{8,13,14}$ in hybrid organic devices, the spin injection/extraction is the key factor and proper design of the OSC/FM electrode interface, also called spinterface, is thus crucial. ${ }^{15,16}$ The efficacy of a spinterface is mainly based on two properties:

(i) The energy level alignment. The spin injection/extraction is carried out by electron/hole injection which has been widely studied for decades in the field of organic electronics. It is well known that the interfacial energy level alignment is a dominant factor. ${ }^{17,18}$ The barrier formed between Fermi level of the metal and the lowest unoccupied molecular orbital (LUMO) or highest occupied molecular orbital (HOMO) of the molecule needs to be overcome for the carrier injection. An optimized energy level alignment will facilitate the carrier injection and also the spin injection/extraction.

(ii) The magnetic properties of FM/OSC interfaces. There are many reports on the interfacial spin-polarization of organic molecules adsorbed on FM films, ${ }^{19-22}$ which is believed to arise from orbital hybridization between OSC and FM atoms. On the other hand, the magnetic property of the FM is also influenced by the contacting OSC. ${ }^{21,23-25}$ This reactive modification effect of organic molecules on the magnetic property of FM metals is likely to play a significant role in the property of organic spin valve device as well. Therefore, the design and study of spinterfaces should take all of these points into consideration simultaneously, i.e., the energy level alignment, possible spin-polarization of the interface OSC and possible modification of the interface FM magnetic properties. Though such comprehensive data can be pieced together for a few FM/OSC systems from results of several different studies, we will here present the "full" analysis for two prototypical FM/OSC systems used to obtain high work function and low work function spin injecting electrodes, respectively.

In this study, we use iron ( $\mathrm{Fe})$ as the FM bottom electrode. Two typical organic molecules, tetracyanoquinodimethane (TCNQ) and acridine orange base (AOB) are chosen to form the spinterface with Fe. TCNQ and AOB are two different types of molecules in which the former is 
electron acceptor and the latter is electron donor. We choose the two molecules as prototypes for inducing opposite energy level alignment optimizing effects. In previous studies, the two molecules have been used to tune the vacuum level of substrate to optimize the interfacial energy alignment. For instance, in F. Li's work, ${ }^{26}$ AOB could reduce the work function of $\mathrm{Au}$ to make it a good cathode for $\mathrm{C}_{60}$-based organic solar cells. Considering that $\mathrm{C}_{60}$ is also a very promising spin-transport material that has already been used in room-temperature organic spin valve, ${ }^{27}$ it is reasonable to infer that TCNQ and AOB are the potential buffer layer materials that could also be utilized in the organic spin valves. Ultra-violet photoelectron spectroscopy (UPS) experiments are carried out to investigate the energy level alignment while near edge X-ray absorption fine structure (NEXAFS) and X-ray magnetic circular dichroism (XMCD) to characterize the magnetic property of OSC/Fe interface. Semiquantitative calculations based on XMCD sum rules ${ }^{28,29}$ is used to reveal the detailed influence of the two types of adsorbed organic molecules on Fe substrate. Our results reveal the necessity of investigating the magnetic property changes of both the OSC and the FM during the process of energy level alignment engineering.

\section{Experimental methods}

The UPS experiments were performed using a Scienta ESCA 200 spectrometer connected to a preparation chamber. The analysis thus could be carried out on samples prepared in situ. The base pressure of spectrometer and preparation chamber was $10^{-10}$ mbar and $10^{-9}$ mbar, respectively. A He I (21.2 eV) gas discharge lamp worked as the ultraviolet light source and a bias of $-3.0 \mathrm{~V}$ was applied on the sample holder for the clear observation of the low-energy secondary electron cutoff. Thin films of Fe were deposited on gold-coated silicon substrate using an UHV e-beam evaporator mounted in the preparation chamber. TCNQ and AOB were deposited by thermal evaporation from a differentially pumped Knudsen cell system, inserted into the preparation chamber after chamber-baking to avoid contamination due to the low sublimation temperature of the molecules. The measurement sequence involved characterization of the bare Fe substrates and subsequent stepwise deposition and characterization of molecular add-layers.

NEXAFS and XMCD measurements were performed at beamline D1011 of the MAX-II storage ring, located at the MAX-Laboratory for Synchrotron Radiation Research in Lund, Sweden. Thin films of $\mathrm{Fe}$ were deposited in $\sim$ submonolayer steps onto Si-substrates with a native oxide using an UHV e-beam evaporator mounted in the preparation chamber. XMCD measurements were carried out after each deposition step and deposition was halted when the films showed a noticeable Fe $L$-edge XMCD signal. TCNQ and $\mathrm{AOB}$ were sublimated with a simple home-made Knudsen cell mounted prior to baking. The chamber-baking temperature thus was specially lowered to avoid affecting the two organic materials. The growth rates of TCNQ and AOB are estimated by the pre-calibrated source current and pressure, monitored by NEXAFS.

\section{Ultraviolet photoelectron spectroscopy (UPS)}

Figure 1 shows the UPS spectra of TCNQ deposited on Fe. The TCNQ thickness increases from bottom to top as indicated by the arrows in Figure $1 \mathrm{a}$ and $1 \mathrm{~b}$. Interfacial states are evident with a gap-state forming in the region between $\sim 2.5 \mathrm{eV}$ and the Fermi level for very thin TCNQ

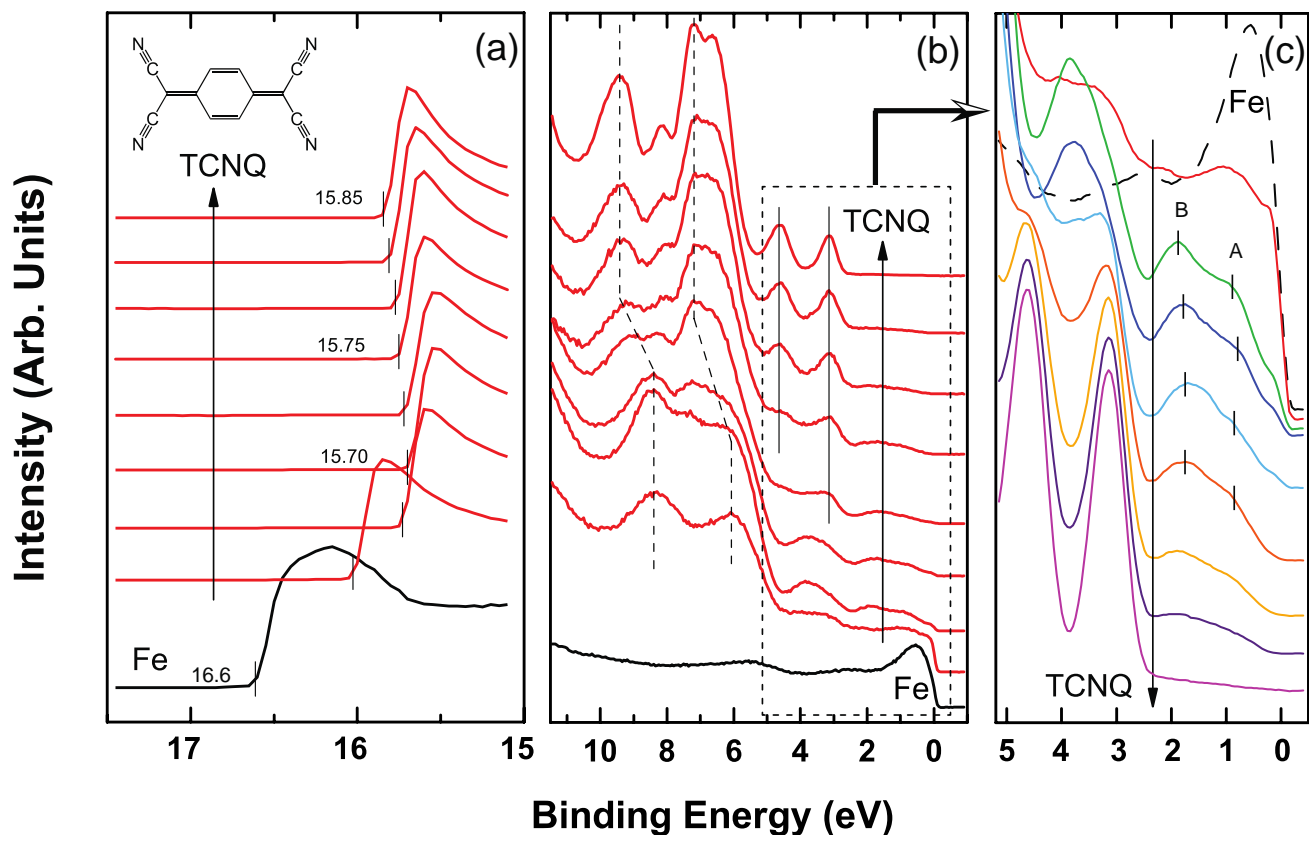

Figure 1. (a) and (b) The UPS spectra of Fe substrate and subsequently deposited TCNQ (red line). The thickness of TCNQ is increasing from the bottom up. (c) The close-up of the frontier valence region. The thickness of TCNQ is increasing inversely from top to bottom for the clearer observation. 


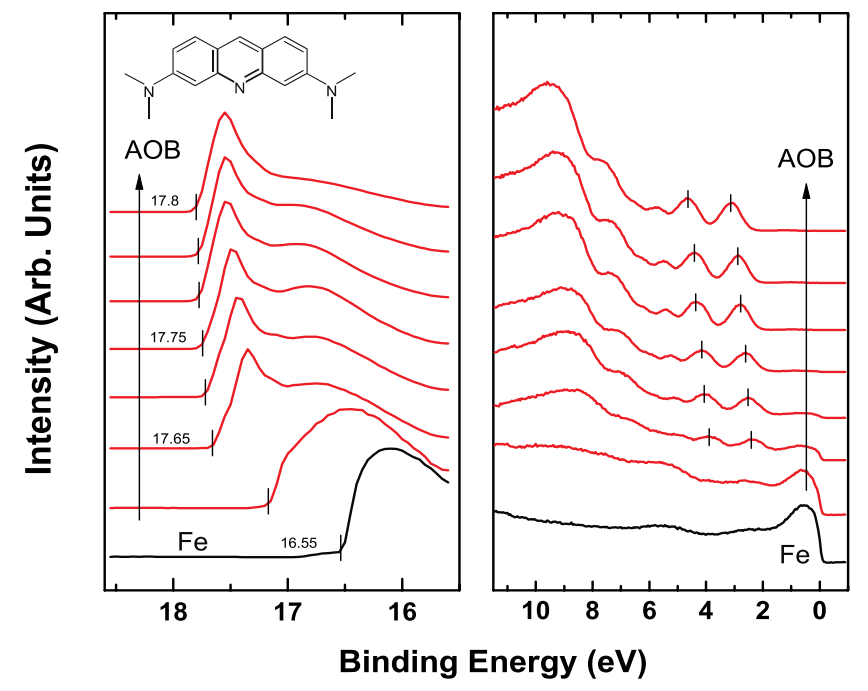

Figure 2. The UPS spectra of Fe substrate and subsequently deposited AOB (red line). The thickness of AOB is increasing from the bottom up.

films, i.e., in the initial stage of TCNQ adsorption. This is not surprising when considering the strong acceptor nature of TCNQ and the reactive nature of atomically clean Fe surface. A blow-up of the frontier valence region is shown in Figure 1c. It can be readily seen that the frontier region features two main peaks, denoted by $\mathrm{A}$ and $\mathrm{B}$ and there is a small shoulder at $\sim 0.2 \mathrm{eV}$. The spectrum in the gap region is found to be quite similar to the valence orbital spectrum of $\mathrm{K}^{+}(\mathrm{TCNQ})^{-30}$ and for TCNQ monolayers on Ni(111), from which we assign the B-feature to the destabilized pristine HOMO and the A-feature to the $\sim$ singly populated pristine LUMO. Besides the formation of the gap-states, the frontier occupied electronic structure is also quite different from that in the bulk TCNQ film, and the features are broader. This suggests charge transfer from the iron substrate to the TCNQ molecules through hybridization of $\pi$ bond orbitals with the $\mathrm{Fe} 3 \mathrm{~d}$ atomic orbitals, rather than integer charge transfer by tunneling, which is reasonable given the reactive nature of the clean Fe surface. The deeper lying main peaks are located at lower binding energy compared with the corresponding features of the bulk layer, as indicated by the two dash lines. Considering that the TCNQ molecule contacting with Fe atom is probably bent from the original planar structure due to the bonding interaction with the substrate, the possible change of molecular shape may also influence the electron orbital. ${ }^{32,33}$ With increasing TCNQ coverage, two separate peaks form as the feature of bulk TCNQ in the frontier valence region at the position of $3.2 \mathrm{eV}$ and $4.7 \mathrm{eV}$ below the Fermi level, separately, in agreement with the values reported in literature. $^{31,32}$ The evolution of the work function is also governed by the interface formation. From the so-called secondary electron cutoff the work function of Fe can be determined as $\sim 4.6 \mathrm{eV}$, corresponding to the typical value in literature. ${ }^{19}$ Once TCNQ molecules adsorb on Fe to form the interface, an interface dipole appears, achieving a maximum value of $\sim 0.9 \mathrm{eV}$. Consequently the work function

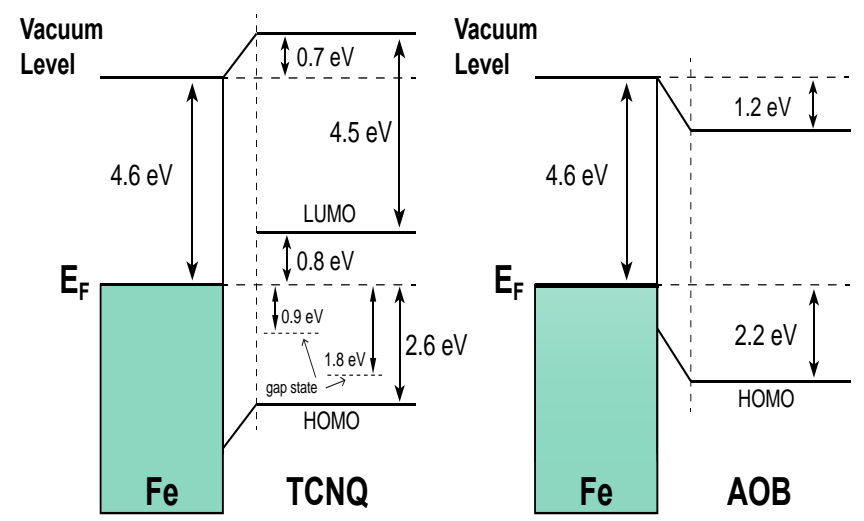

Figure 3. Energy level alignments of $\mathrm{TNCQ} / \mathrm{Fe}$ and $\mathrm{AOB} / \mathrm{Fe}$ interfaces derived from UPS results.

increases to higher values and reaches a value of $\sim 5.5 \mathrm{eV}$ very quickly, when TCNQ is still a submonolayer. Previous research has shown that the work function of organic semiconductor molecules chemisorbed on metals is closely correlated to the orientation of the chemisorbed molecules. ${ }^{34}$ In view of the relatively high work function of TCNQ/Fe interface $(\sim 5.5 \mathrm{eV})$ in present studies, it is believed that the molecular plane of adsorbed TCNQ is parallel to Fe substrate, i.e., TNCQ molecules "lay" on the substrate in the interface formation. However, as TCNQ deposition goes on, the work function decreases, by about $0.2 \mathrm{eV}$. The decrease of work function implies that the subsequent deposition of TCNQ molecules may lead to a depolarization effect, which arises from the dipole-dipole interaction as TCNQ goes from submonolayer to monolayer. ${ }^{35,36}$ It could also be caused by a change in the molecular orientation as the surface is saturated, which in turn affects the work function, similar to the case reported for hexaazatriphenylenehexacarbonitrile molecules chemisorbed on $\operatorname{Ag}(111){ }^{37}$

The situation for AOB deposited on Fe, presented in Figure 2, is quite different compared with that of TCNQ. No hybrid state appears at the molecule/Fe interface and the frontier occupied electronic structure of the monolayer is the same as that of the bulk, showing the interaction between AOB and Fe is not as strong as that of TCNQ and Fe, but rather likely undergo physisorption. With increasing coverage of $\mathrm{AOB}$, the valence band features become clear. Similar to that of TCNQ, the frontier features of AOB contain two separate peaks, at the position of $2.4 \mathrm{eV}$ and $4 \mathrm{eV}$ below Fermi level, separately. As the AOB thickness increases, the whole spectrum moves slightly to higher binding energy, in line with previous reports on AOB physisorbed on $\mathrm{Au}^{26}$

The energy level alignment of the spinterface derived from the data of UPS spectra is shown in Figure 3. The work function of $\mathrm{Fe}$ is $4.6 \mathrm{eV}$. For TCNQ on Fe, the interface dipole is $+0.7 \mathrm{eV}$ (full coverage) and the leading edge of HOMO peak is located at $2.6 \mathrm{eV}$ below $\mathrm{E}_{\mathrm{F}}$. The vertical ionization energy (IE), which refers to the energy difference between $\mathrm{E}_{\mathrm{vac}}$ and the HOMO, is $7.9 \mathrm{eV}$ in good agreement with previous literature data. ${ }^{38}$ According to 
(a)

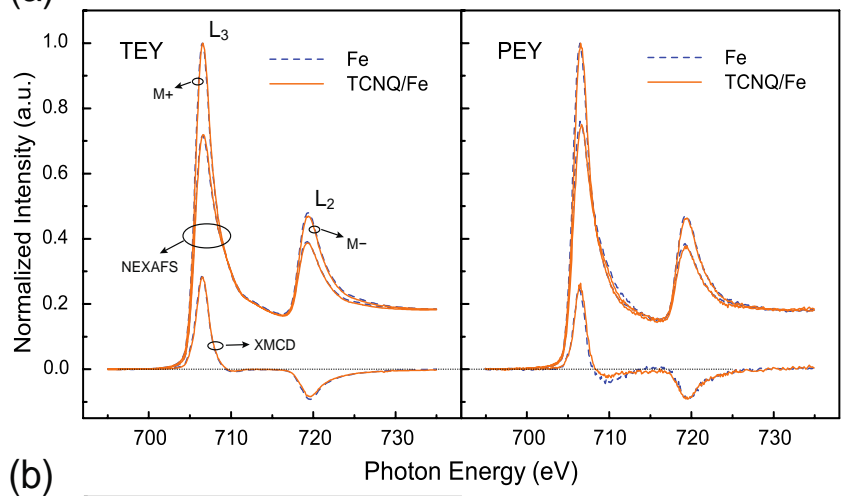

(b)

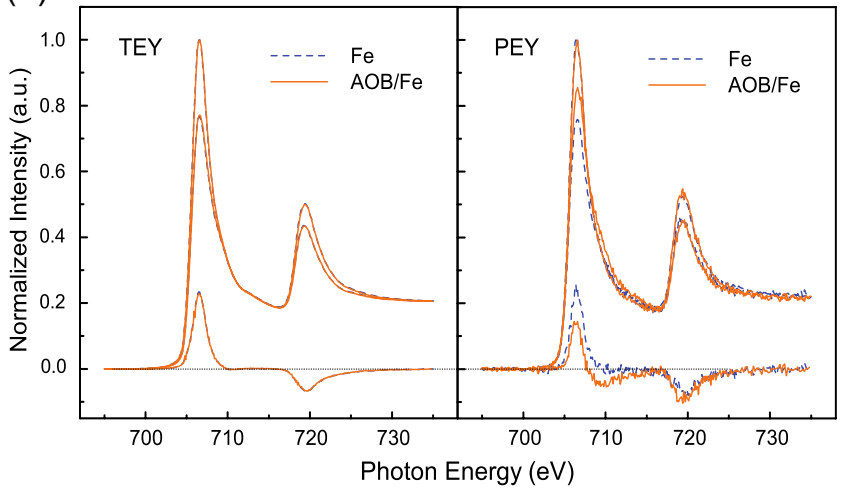

Figure 4. Fe L-edge NEXAFS and XMCD spectra of Fe substrate before (blue) and after (brown) the molecule adsorption. (a) TCNQ molecule. (b) AOB molecule. The left side is TEY mode and the right side is PEY mode.

literature, ${ }^{39,40}$ the LUMO is located at $4.5 \mathrm{eV}$ below $\mathrm{E}_{\mathrm{vac}}$ and the value is also known as electron affinity (EA). Therefore, the injection barriers of electron and hole from Fe to TCNQ are $0.8 \mathrm{eV}$ and $2.6 \mathrm{eV}$, respectively. For AOB on $\mathrm{Fe}$, the interface dipole is $-1.2 \mathrm{eV}$ and the leading edge of HOMO peak is located at $2.2 \mathrm{eV}$ below $\mathrm{E}_{\mathrm{F}}$. The IE of AOB is $5.6 \mathrm{eV}$ while the EA is unknown for the lack of existing data of LUMO. According to the work function tuning effect of both TCNQ and AOB, it can be inferred that a $\sim$ monolayer of TCNQ could enhance the hole injection properties of a $\mathrm{Fe}$-anode (by significantly increasing the work function) while a $~$ monolayer of AOB could facilitate the electron injection of a Fe-cathode (by significantly decreasing the work function).

\section{X-ray magnetic circular dichroism (XMCD)}

The energy level alignment is critical from the point view of charge injection. For spin injection, also the spin polarization accompanied with the charge is indispensable. Therefore, the interfacial magnetic property influenced by the molecules is essential to evaluate if a particular ad-layer is suitable for spin injection. The FM electrode itself is an efficient spin injector. At the interface, however, several outer atomic layers may be influenced by the adsorbed organic molecules. ${ }^{41,42}$ The XMCD measurement is a powerful technique that can reveal the magnetic property of samples, which is obtained by subtracting the two spectra of NEXAFS for parallel $(\mathrm{M}+)$ and antiparallel $\left(\mathrm{M}^{-}\right)$alignment

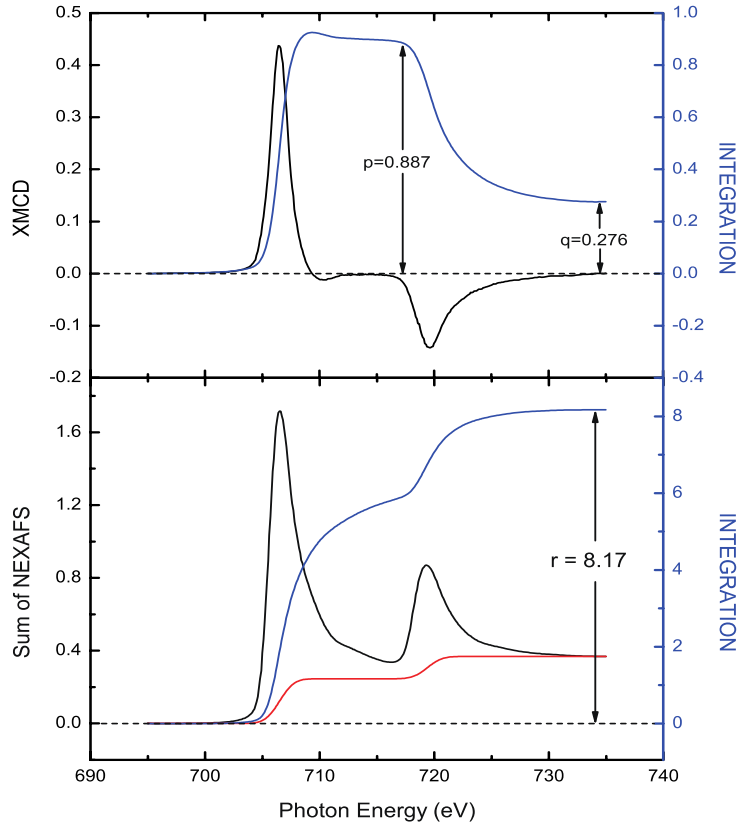

Figure 5. The acquisition method of the parameter $p, q$ and $r$ that is involved in the calculation of magnetic moment based on sum rule (taking the TEY spectra of Fe before TCNQ deposition for instance and the XMCD spectrum being corrected by multiplying by the factor).

between the magnetization and photon helicity. Here we analyze the Fe $L_{2,3}$-edge XMCD spectra before and after TCNQ (or AOB) deposition, separately, to investigate the effects of molecule adsorption. The probing depth of $\mathrm{XMCD}$ is usually 5-6 nanometers or even more, causing the signal coming from lower bulk $\mathrm{Fe}$ atoms to partially obscure the interfacial effects, hence the use of thin Fe films in our study to reduce bulk contribution. Furthermore, we sue two different modes for the XMCD measurement: total electron yield (TEY) and partial electron yield (PEY). The difference lies in that for PEY mode we apply an additional retarding voltage which will filter out the low-energy photoelectron and hence make the measurement more surface sensitive. XMCD spectra derived from PEY mode thus reflect more the surface magnetic information, i.e. from the $\mathrm{OSC} / \mathrm{Fe}$ interface, than those obtained from TEY mode.

Figure 4 shows the XMCD spectra of Fe $L_{2,3}$-edge of the same sample before and after the deposition of organic molecular monolayer, denoted by blue dotted line and brown solid line, respectively. The spectra are normalized to the $L_{3}$-peak height of the NEXAFS spectra $(\mathrm{M}+)$. The influence of molecular adsorption can be reflected by contrasting the two XMCD spectra before and after that. It can be seen in Figure 4a that TEY XMCD spectra have almost no change before and after TCNQ deposition. It is reasonable considering the signal reflecting the magnetic property of bulk $\mathrm{Fe}$ which should not be much affected by the TCNQ overlayer. Then PEY XMCD measurement is utilized to reveal the magnetic property of Fe closer to the interface. Because of the retarding voltage and the resulting weaker signal, PEY spectra have a worse signal-to-noise ratio than TEY spectra. From the right side of Figure $4 a$, it can be seen that just 
Table 1. Summary of the magnetic moment of Fe derived from the XMCD sum rules

\begin{tabular}{lcccccc}
\hline \hline & $\boldsymbol{p}$ & $\boldsymbol{q}$ & $\boldsymbol{r}$ & $\boldsymbol{m}_{\text {orb }}\left(\boldsymbol{\mu}_{\mathrm{B}}\right)$ & $\boldsymbol{m}_{\text {spin }}\left(\boldsymbol{\mu}_{\mathrm{B}}\right)$ & $\boldsymbol{r a t i o}$ \\
\hline Fe before TCNQ(bulk) & 0.887 & 0.276 & 8.17 & 0.152 & 1.75 & 0.087 \\
with TCNQ (bulk) & 0.875 & 0.285 & 8.03 & 0.160 & 1.73 & 1.33 \\
Fe before AOB(bulk) & 0.773 & 0.311 & 8.66 & 0.162 & 0.092 \\
with AOB (bulk) & 0.767 & 0.317 & 8.59 & 0.167 & 0.122 & 0.127 \\
\hline Fe before TCNQ(surface) & 0.570 & 0.015 & 7.95 & 0.009 & 1.43 & 0.006 \\
with TCNQ (surface) & 0.523 & -0.046 & 7.52 & -0.028 & -0.019 \\
Fe before AOB(surface) & 0.662 & 0.169 & 8.18 & 0.094 & 1.50 & 1.37 \\
with AOB (surface) & 0.009 & -0.585 & 8.90 & -0.297 & 0.91 & -0.326 \\
\hline \hline
\end{tabular}

Bulk and surface refer to the results measured in TEY and PEY modes, respectively.

a slight difference emerges at the extensive region of $L_{3}$ edge, indicating a near negligible effect on the spin polarization of Fe induced by the TCNQ deposition. Figure 4b depicts the case of AOB/Fe. Very similar to that of TCNQ, the TEY spectra show the identical results before and after AOB deposition, as it is expected for the weaker interaction between AOB molecules and Fe atoms. However, for PEY XMCD spectra, we observe a noticeable difference after the deposition of the AOB overlayer. The XMCD signal of the Fe with an AOB overlayer is reduced in intensity by $40 \%$ at $L_{3}$-edge, while the overall size at $L_{2}$-edge is increased. Since the measurement is more sensitive to the interface, this result means a changed magnetic moment of $\mathrm{Fe}$ atoms in the surface region when covered by an AOB overlayer. To obtain more detailed information, the XMCD sum rules are applied to make semiquantitative calculations. The method described in ref. 29 is utilized to calculate the magnetic moment of Fe. We take the TEY spectra of Fe before TCNQ deposition for instance, as shown in Figure 5. The values of $p$ and $q$ are derived from the integration of $\mathrm{XMCD}$, and the value of $r$ is derived from the integration of the sum of the two NEXAFS spectra after subtracting the two-step-like background (see ref. 28, 29 for detailed theoretical analysis). Based on the obtained $p$ and $q$, we get $m_{\text {spin }}=(6 p-4 q)\left(10-n_{3 \mathrm{~d}}\right) / r, m_{\mathrm{orb}}=4 q\left(10-n_{3 \mathrm{~d}}\right) / 3 r\left(n_{3 \mathrm{~d}}=6.61\right.$ for $\mathrm{Fe}$, the same value as in ref. 29). It is necessary to take into account the incident angle $\left(30^{\circ}\right.$ with respect to the sample surface) and the degree of circular polarization (75\%), as described in ref. 29,43. The XMCD spectra (also the values of $p$ and $q$ ) thus are corrected by multiplying by a factor of $\left(1 / \cos 30^{\circ}\right) / 0.75$, corresponding to the parallel incidence of fully circular polarized light, while the sum spectra of NEXAFS (also the value of $r$ ) do not change. All of the results are summarized in Table 1

. Looking first at the bare Fe data, it is clear that the magnetic moments of $\mathrm{Fe}$ thin layers derived from PEY are different from those derived from TEY, especially for

the orbital moment, suggesting that the surface layer magnetic moment is different from the bulk. It is also noticeable that the magnetic moments of different bare Fe substrates differ somewhat in our study. We assign this phenomenon to arise from the amorphous nature of the Fe films used. However, what counts is the variation of XMCD spectra before and after the molecular adsorption. As mentioned above, there is no significant change on the TEY results upon OSC coverage, which reflect more bulk information, before and after molecule deposition. For both TCNQ and AOB molecules, the values of $m_{\text {orb }}$ and $m_{\text {spin }}$ just have a very slight change which is in the range of measurement and data processing error. By contrast, the PEY results are much more complicated. For the TCNQ-modified Fe surface, the spin moment of $\mathrm{Fe}$ changes from $1.43 \mu_{\mathrm{B}}$ to 1.50 $\mu_{\mathrm{B}}$, and the orbital moment changes from $0.009 \mu_{\mathrm{B}}$ to $-0.028 \mu_{\mathrm{B}}$. For the AOB-modified Fe surface, a significantly stronger change in $\mathrm{Fe}$ magnetization is observed. The spin moment of Fe changes from $1.37 \mu_{\mathrm{B}}$ to $0.91 \mu_{\mathrm{B}}$, decreasing by as much as $\sim 0.46 \mu_{\mathrm{B}}$. The orbital moment changes from $0.094 \mu_{\mathrm{B}}$ to $-0.297 \mu_{\mathrm{B}}$. The physisorbed AOB molecules thus have a significant effect on the magnetization of Fe substrate. By contrast, TCNQ molecules affect the magnetization of Fe substrate much less, despite chemisorbing onto Fe. According to the XMCD results, it can be inferred that TCNQ molecule has little influence on the spin polarization of $\mathrm{Fe}$ interface, while $\mathrm{AOB}$ molecule reduces the interfacial spin polarization of $\mathrm{Fe}$ significantly. Therefore, when TCNQ and AOB molecule are used as buffer layer, the latter's influence on the spin polarization of FM electrode needs to be taken into account, which for $\mathrm{Fe}$ seems to be detrimental.

Also noteworthy is that the orbital moment derived from PEY mode turns to minus value after both TCNQ and AOB deposition, see Table 1. It indicates that the molecules may modify the electron orbital of surface Fe layer, resulting in the peculiar phenomenon that the orbital moment is reversed to an opposite direction to the spin moment. This phenomenon, though likely not critical to spin injection properties since the magnetization of $\mathrm{Fe}$ is dominated by spin moment, is interesting and deserves further confirmation and investigation.

XMCD studies on the $K$-edge of $\mathrm{C}$ and $\mathrm{N}$ were carried out to probe the possible hybridization-induced magnetization of TCNQ and AOB (not shown). No clear XMCD signal was observed in our experiments, however, in spite of the existence of the strong hybrid state with Fe in the case of TCNQ. This result implies that the $\pi$-bond orbitals hybridized with $3 \mathrm{~d}$ orbitals, though essential, does not inevitably lead to the hybridization-induced spin polarization. Besides as the buffer layer, TCNQ and AOB molecules can 
be considered as the candidate of the thin spacer layer in tunneling magnetic junction for which the device performance is dominated by the energy alignment at the FM/OSC interface.

\section{Conclusions}

UPS and XMCD are utilized to investigate the energy level alignment and spin polarization at the spinterface of Fe substrate and TCNQ/AOB molecule adlayers. UPS spectra demonstrate the coexistence of the singly charged negative state and the strong hybrid state at the TCNQ/Fe interface and the physisorption characteristic of $\mathrm{AOB} / \mathrm{Fe}$ interface. According to the work function tuning effect of both TCNQ and AOB on Fe substrate, it can be inferred that TCNQ could facilitate enhanced hole-injection of a $\mathrm{Fe}$ anode while AOB could facilitate enhanced electron injection of a Fe-cathode. According to the XMCD results, TCNQ adlayers have little influence on the spin polarization of Fe interface while, in contrast, AOB adlayers reduce the interfacial spin polarization of Fe significantly. It means that for AOB molecules, the influence on the spin polarization of FM electrode needs to be taken into account, which may lead to a negative effect on overall spin injection despite improved energy level matching. The spin polarization of the organic molecules, TCNQ and AOB, is not observed in our experiment. The results are relevant to the design of organic spin valve device, especially to the selection of the organic buffer layer.

\section{Acknowledgments}

This research is financially supported by the project NMP3-SL-2011-263104-HINTS and the Swedish Research Council (VR) grant 2011-7307.

\section{* Corresponding authors.}

Tel.: +461328 4694 (Z. Sun), +86 2155664725 (Y. Zhan).

E-mail address: zhsun@ifm.liu.se (Z.Sun), yqzhan@fudan.edu.cn (Y. Zhan).

\section{References}

(1) Dediu, V.; Murgia, M.; Matacotta, F. C.; Taliani, C.; Barbanera, S. Solid State Commun. 2002, 122, 181-184.

(2) Xiong, Z. H.; Wu, D.; Vardeny, Z. V.; Shi, J. Nature 2004, 427, 821-824.

(3) Francis, T. L.; Mermer, Ö.; Veeraraghavan, G.; Wohlgenannt, M. New J. Phys. 2004, 6, 185-185.

(4) Ding, B. F.; Yao, Y.; Sun, Z. Y.; Wu, C. Q.; Gao, X. D.; Wang, Z. J.; Ding, X. M.; Choy, W. C. H.; Hou, X. Y. Appl. Phys. Lett. 2010, 97, 163302

(5) Ding, B.; Yao, Y.; Sun, X.; Gao, X.; Xie, Z.; Sun, Z.; Wang, Z.; Ding, X.; Wu, Y.; Jin, X.; Choy, W.; Wu, C.-Q.; Hou, X. Phys. Rev. B 2010, 82, 1-6.

(6) Hu, B.; Yan, L.; Shao, M. Adv. Mater. 2009, 21, 1500-1516.

(7) Wang, F. J.; Xiong, Z. H.; Wu, D.; Shi, J.; Vardeny, Z. V. Synth. Met. 2005, 155, 172-175.

(8) Petta, J.; Slater, S.; Ralph, D. Phys. Rev. Lett. 2004, 93, 136601.

(9) Drew, A. J.; Hoppler, J.; Schulz, L.; Pratt, F. L.; Desai, P.; Shakya, P.; Kreouzis, T.; Gillin, W. P.; Suter, A.; Morley, N. a; Malik, V. K.; Dubroka, A.; Kim, K. W.; Bouyanfif, H.; Bourqui, F.; Bernhard, C.;
Scheuermann, R.; Nieuwenhuys, G. J.; Prokscha, T.; Morenzoni, E. Nat. Mater. 2009, 8, 109-114.

(10) Dediu, V. A.; Hueso, L. E.; Bergenti, I.; Taliani, C. Nat. Mater. 2009, 8, 707-716.

(11) Wang, F.; Vardeny, Z. V. J. Mater. Chem. 2009, 19, 1685.

(12) Dediu, V.; Hueso, L.; Bergenti, I.; Riminucci, A.; Borgatti, F.; Graziosi, P.; Newby, C.; Casoli, F.; De Jong, M.; Taliani, C.; Zhan, Y. Phys. Rev. B 2008, 78, 115203.

(13) Santos, T. S.; Lee, J. S.; Migdal, P.; Lekshmi, I. C.; Satpati, B.; Moodera, J. S. Phys. Rev. Lett. 2007, 98, 016601.

(14) Shim, J.; Raman, K.; Park, Y.; Santos, T.; Miao, G.; Satpati, B.; Moodera, J. Phys. Rev. Lett. 2008, 100, 1-4.

(15) Schulz, L.; Nuccio, L.; Willis, M.; Desai, P.; Shakya, P.; Kreouzis, T.; Malik, V. K.; Bernhard, C.; Pratt, F. L.; Morley, N. a; Suter, A.; Nieuwenhuys, G. J.; Prokscha, T.; Morenzoni, E.; Gillin, W. P.; Drew, a J. Nat. Mater. 2011, 10, 39-44.

(16) Zhan, Y.; Fahlman, M. J. Polym. Sci. Part B Polym. Phys. 2012, 50, 1453-1462.

(17) Ishii, H.; Sugiyama, K.; Ito, E.; Seki, K. Adv. Mater. 1999, 11, 605-625.

(18) Braun, S.; Salaneck, W. R.; Fahlman, M. Adv. Mater. 2009, 21, $1450-1472$

(19) Zhan, Y.; Holmström, E.; Lizárraga, R.; Eriksson, O.; Liu, X.; Li, F.; Carlegrim, E.; Stafström, S.; Fahlman, M. Adv. Mater. 2010, 22, $1626-1630$

(20) Weser, M.; Rehder, Y.; Horn, K.; Sicot, M.; Fonin, M.; Preobrajenski, a. B.; Voloshina, E. N.; Goering, E.; Dedkov, Y. S. Appl. Phys. Lett. 2010, 96, 012504.

(21) Tran, T. L. a.; Wong, P. K. J.; de Jong, M. P.; van der Wiel, W. G.; Zhan, Y. Q.; Fahlman, M. Appl. Phys. Lett. 2011, 98, 222505.

(22) Wong, P. K. J.; Zhang, W.; Wang, K.; van der Laan, G.; Xu, Y.; van der Wiel, W. G.; de Jong, M. P. J. Mater. Chem. C 2013, 1, 1197.

(23) Pernechele, C.; Bergenti, I.; Solzi, M.; Ghidini, M.; Casoli, F.; Dediu, V. J. Magn. Magn. Mater. 2010, 322, 1251-1254.

(24) Zhang, X.; Mizukami, S.; Kubota, T.; Oogane, M.; Naganuma, H.; Ando, Y.; Miyazaki, T. Appl. Phys. Lett. 2011, 99, 162509.

(25) Zhan, Y. Q.; Liu, X. J.; Carlegrim, E.; Li, F. H.; Bergenti, I.; Graziosi, P.; Dediu, V.; Fahlman, M. Appl. Phys. Lett. 2009, 94, 053301 .

(26) Li, F.; Zhou, Y.; Zhang, F.; Liu, X.; Zhan, Y.; Fahlman, M. Chem. Mater. 2009, 21, 2798-2802.

(27) Gobbi, M.; Golmar, F.; Llopis, R.; Casanova, F.; Hueso, L. E. Adv. Mater. 2011, 23, 1609-1613.

(28) Thole, B.; Carra, P.; Sette, F.; van der Laan, G. Phys. Rev. Lett. 1992, 68, 1943-1946.

(29) Chen, C.; Idzerda, Y.; Lin, H.; Smith, N. Phys. Rev. Lett. 1995, $75,152-155$.

(30) Grobman, W.; Pollak, R.; Eastman, D. Phys. Rev. Lett. 1974, 32, 534-537.

(31) Giergiel, J.; Wells, S.; Land, T. a.; Hemminger, J. C. Surf. Sci. 1991, 255, 31-40.

(32) Medjanik, K.; Perkert, S.; Naghavi, S.; Rudloff, M.; Solovyeva, V.; Chercka, D.; Huth, M.; Nepijko, S.; Methfessel, T.; Felser, C.; Baumgarten, M.; Müllen, K.; Elmers, H.; Schönhense, G. Phys. Rev. B 2010, 82, 245419.

(33) Rangger, G.; Hofmann, O.; Romaner, L.; Heimel, G.; Bröker, B.; Blum, R.-P.; Johnson, R.; Koch, N.; Zojer, E. Phys. Rev. B 2009, 79, 165306.

(34) Duhm, S.; Heimel, G.; Salzmann, I.; Glowatzki, H.; Johnson, R. L.; Vollmer, A.; Rabe, J. P.; Koch, N. Nat. Mater. 2008, 7, 326-332.

(35) Vager, Z.; Naaman, R. Chem. Phys. 2002, 281, 305-309. 
(36) Cornil, D.; Olivier, Y.; Geskin, V.; Cornil, J. Adv. Funct. Mater. 2007, 17, 1143-1148.

(37) Bröker, B.; Hofmann, O. T.; Rangger, G. M.; Frank, P.; Blum, R.-P.; Rieger, R.; Venema, L.; Vollmer, a.; Müllen, K.; Rabe, J. P.; Winkler, a.; Rudolf, P.; Zojer, E.; Koch, N. Phys. Rev. Lett. 2010, 104, 246805.

(38) Kahn, A.; Koch, N.; Gao, W. J. Polym. Sci. Part B Polym. Phys. 2003, 41, 2529-2548.

(39) Braun, S.; Liu, X.; Salaneck, W. R.; Fahlman, M. Org. Electron. 2010, 11, 212-217.

(40) Mandoc, M. M.; Kooistra, F. B.; Hummelen, J. C.; de Boer, B.; Blom, P. W. M. Appl. Phys. Lett. 2007, 91, 263505.

(41) Bertoni, G.; Calmels, L.; Altibelli, A.; Serin, V. Phys. Rev. B 2005, 71, 075402.

(42) Atodiresei, N.; Brede, J.; Lazić, P.; Caciuc, V.; Hoffmann, G.; Wiesendanger, R.; Blügel, S. Phys. Rev. Lett. 2010, 105, 066601.

(43) Tran, T. L. A.; Cakır, D.; Wong, P. K. J.; Preobrajenski, A. B.; Brocks, G.; van der Wiel, W. G.; de Jong, M. P. ACS Appl. Mater. Interfaces 2013, 5, 837-841. 